This is the peer reviewed version of the following article: Domínguez, J., Fernández-Nieto, F., Brea, J., Catto, M., Paleo, M., \& Porto, S. et al. (2016). 8-Aminomethyl-7-hydroxy-4-methylcoumarins as Multitarget Leads for Alzheimer's Disease. Chemistryselect, 1(11), 2742-2749, which has been published in final form at https://doi.org/10.1002/slct.201600735.

This article may be used for non-commercial purposes in accordance with Wiley Terms and Conditions for Use of Self-Archived Versions 


\title{
8-Aminomethyl-7-hydroxy-4-methylcoumarins as Multitarget Leads for Alzheimer's Disease
}

\author{
José L. Domínguez, ${ }^{[\mathrm{a}]}$ Fernando Fernández-Nieto, ${ }^{[\mathrm{b}]}{ }$ José M. Brea, ${ }^{[\mathrm{c}]}$ Marco Catto, ${ }^{[\mathrm{d}]} \mathrm{M}$. Rita Paleo, ${ }^{[\mathrm{b}]}$ \\ Silvia Porto, ${ }^{[\mathrm{b}]}$ F. Javier Sardina,${ }^{[\mathrm{b}]}$ Marian Castro, ${ }^{[\mathrm{c}]}$ Leonardo Pisani, ${ }^{[\mathrm{d}]}$ Angelo Carotti, ${ }^{[\mathrm{dd}]}$ Ramón Soto- \\ Otero, ${ }^{[\mathrm{e}]}$ Estefanía Méndez-Alvarez, ${ }^{[\mathrm{e}]} \mathrm{M}$. Carmen Villaverde, ${ }^{*[\mathrm{a}]}$ and Fredy Sussman ${ }^{*[\mathrm{a}]}$
}

[a] Dr. J. L. Domínguez, Prof. M. C. Villaverde, Dr. F. Sussman

Departamento de Química Orgánica, Facultad de Química,

Universidade de Santiago de Compostela, 15782 Santiago de Compostela (Spain)

E-mail: fredy.sussman@usc.es

[b] Dr. F. Fernández-Nieto, Prof. M. R. Paleo, Dr. S. Porto, Prof. F. J. Sardina

Departamento de Química Orgánica and Centro Singular de Investigación en Química Biolóxica e Materiais Moleculares (CIQUS)

Universidade de Santiago de Compostela, 15782 Santiago de Compostela (Spain)

[c] Dr. J. M. Brea, Prof. M. Castro

Departamento de Farmacología, Instituto de Farmacia Industrial, Centro de Investigación en Medicina Molecular y Enfermedades Crónicas (CIMUS), Universidade de Santiago de Compostela, 15782 Santiago de Compostela (Spain)

[d] Dr. M. Catto, Dr. L. Pisani, Prof. A. Carotti

Dipartimento di Farmacia-Scienze del Farmaco,

Università degli Studi di Bari "Aldo Moro", 70125 Bari (Italy)

[e] Prof. R. Soto-Otero, Prof. E. Méndez-Alvarez

Grupo de Neuroquímica, Departamento de Bioquímica y Biología Molecular, Facultad de Medicina,

Universidade de Santiago de Compostela, 15782 Santiago de Compostela (Spain)

Supporting information for this article is given via a link at the end of the document

\begin{abstract}
This work is part of our ongoing research in the discovery of multitarget therapeutic agents for Alzheimer's disease (AD). A literature screening, based on our recently proposed pharmacophore, led to the identification of 8-aminomethyl-7-hydroxy-4-methyl coumarins as potential multitarget leads for AD. The results of a computer-assisted protocol developed by us to validate multitarget hits for $A D$ indicated that our coumarin candidates were viable leads only for AChE inhibition as later validated by biological assays. The results of $\mathrm{BChE}$ binding and propidium displacement assays indicate that our first generation compounds bind to the PAS site in AChE. We designed new generations of coumarin derivatives with a longer substituent at position 8 aimed at leads with more efficient interaction at the catalytic anionic site (CAS). Inhibition data and docking simulations indicated that an anilino-capping group reached the CAS region of AChE and determined also a higher inhibitory potency towards BChE. The best compound obtained, with a $\mathrm{N}$ benzylpiperidine fragment, displayed sub-micromolar affinity for $\mathrm{AChE}$, affinity for $\mathrm{BChE}$, and precluded $\mathrm{A} \beta$ amyloid aggregation with a potency similar to that of 9,10-anthraquinone, making it a multitarget lead viable for further improvement.
\end{abstract}

\section{Introduction}

Alzheimer disease (AD), a cerebral neurodegenerative pathology that is the main cause of dementia in older people, is characterized by the progressive formation of insoluble amyloid plaques and fibrillary tangles. Despite the enormous efforts carried out by academic institutions and pharmaceutical industries, $A D$ is an illness with unmet medical needs since the only drugs available in clinic (i.e., acetylcholinesterase (AChE) inhibitors and a NMDA receptor antagonist) have symptomatic and palliative effects and do not modify the course of the disease..$^{[1,2]}$
The most exploited hypothesis for the origin of $A D$ is the one related to the amyloid cascade, ${ }^{[3]}$ which singles out low molecular weight aggregates, fibrils and plaques of the amyloid beta peptide ( $A \beta$, a peptide of 40 or 42 residues) as the cause of $A D$, since their presence interrupts the synaptic connections, precludes the right inter-neuron orientation and ultimately leads to neuronal death. ${ }^{[4,5]}$ The $A \beta$ peptides are produced by the hydrolysis of the amyloid precursor protein (APP) by two aspartic proteases, $\gamma$ - and $\beta$-secretase (BACE-1), and the low molecular weight $A \beta$ oligomers have been associated to neurotoxic effects. ${ }^{[6]}$ The last decade has witnessed an all-out effort to discover inhibitors of these two enzymes that could become drug leads for the treatment of $A D$, but all the candidates have failed either at pre-clinical or clinical stages. ${ }^{[1,2]}$ The inhibition of $A \beta$ peptide aggregation has become an important target as well for drug lead discovery, although no $A \beta$ aggregation inhibitor has surpassed the clinical trials either. On the other hand, Inestrosa et al. ${ }^{[7]}$ have shown that the peripheral anionic site (PAS) in AChE could be an additional therapeutic target, since it is a nucleation site for the $A \beta$ peptide aggregation and hence its inhibition could hinder this process. Finally, the inhibitors of AChE could also inhibit butyrylcholinesterase $(\mathrm{BChE})$, and therefore have a bearing on the cholinergic pathway by precluding the hydrolysis of acetylcholine and probably enhancing (albeit temporarily) cognition in $A D$ patients. Furthermore, preventing the neurotoxic effects of reactive oxygen species (ROS) with radical scavengers and/or inhibiting their formation with monoamine oxidase (MAO) inhibitors, which block the catalytic generation by MAO of hydrogen peroxide, a precursor of ROS, could be also an important strategy to tackle $\mathrm{AD} .^{[8,9]}$

The great variety of targets opens the door to a new approach aimed at the discovery of molecules addressing simultaneously multiple targets of $A D$. This novel paradigm, which deviates radically from the traditional "one target-one molecule strategy", has recently received increasing attention. ${ }^{[10-22]}$ The major hurdle 
in the search for multitarget leads lays on the substantial functional and structural differences amongst the targets, an issue that hinders drastically this therapeutic strategy.

Coumarins are natural phytochemicals showing a wide range of pharmacological activities such as hepatoprotective, antiinflammatory, antitumor, antiallergic, antiviral, antifungal, antimicrobial, antiasthmatic, antioxidant, antinociceptive, antidiabetic and antidepressant effects, ${ }^{[23]}$ as well as antiamnesic and memory restorative functions. ${ }^{[24]}$

The potential engagement of the aromatic coumarin ring in hydrophobic, $\pi-\pi$ and $\pi$-cation interactions, and in hydrogen bonding with its lactonic group, makes coumarins efficient ligands of many therapeutically relevant targets. ${ }^{[23-27]}$

Many naturally occurring, as well as chemically synthesised coumarins, have been proposed as potential $A D$ therapeutics, chiefly for their inhibitory activity against AChE and/or BChE. ${ }^{[23,}$ 28-33] Some coumarins act also on other $A D$ related targets like $A \beta$ aggregation $^{[34]}$ and BACE-1. ${ }^{[35,36]}$ The selective, or multiple, activity of coumarins against $A D$ protein targets has been shown to depend on substitution pattern and type of substituents. The recognition of key structural features of coumarin template and substituents for a selective or multiple pharmacological activity has helped in the design and synthesis of new analogues with high potency and selectivity against one target, e.g., AChE, or with a multiple activity resulting from a different combination of AChE, BACE-1 and A $\beta$ aggregation, ${ }^{[14,35]}$ or MAO inhibition. ${ }^{[37]}$ For instance, positions 3,4 and 7 of the coumarin were the most favorable for adding an amino fragment, through a flexible tether to get a dual binding to both PAS and CAS in AChE and to afford inhibitors with high potency and selectivity over BChE. ${ }^{[23]}$ Similar substitutions, mainly at 4 and 7 positions allowed the synthesis of potent and selective MAO-B inhibitors ${ }^{[38-43]}$ and of MAOB-AChE dual inhibitors. ${ }^{[44,45]}$

Recently, we have proposed an in silico procedure for the design of multitarget leads aimed at amyloid cascade and cholinergic pathways in $A D .^{[19]}$ Its first application led us to the discovery of a family of substituted indoles that inhibited $A \beta$ aggregation, BACE-1, BChE and AChE by binding both its catalytic anionic site (CAS) and PAS. ${ }^{[19]}$ The initial step of the protocol relies on a database search of compounds that comply with a pharmacophore proposed by us. One of the features of this template is a hydroxyethylamino group that is of the essence for the specific anchoring of the resulting compounds to the catalytic machinery of BACE-1.

As a result of our database search of hydroxyalkylamino compounds with the structural requirements to interact with the catalytic dyad of BACE-1, we discovered a series of known 8aminomethyl-7-hydroxy coumarins (see Figure 1) in several chemical libraries. The systematic literature exploration led us to identify some Mannich bases of the 7-hydroxy-4-methyl coumarin $(\mathrm{R} "=\mathrm{Me})$ whose analogues exhibited antiviral activity against Flaviviradae. ${ }^{[46]}$

In this work we propose a novel coumarin-based scaffold indicated in Figure 1 for the search of multitarget leads. The results of docking simulations for AChE and BACE-1 as well as molecular dynamics (MD) based simulations for $A \beta$ aggregation predicted that our first generation coumarin derivatives should inhibit only the first of these targets, an outcome that was confirmed later by in vitro assays.

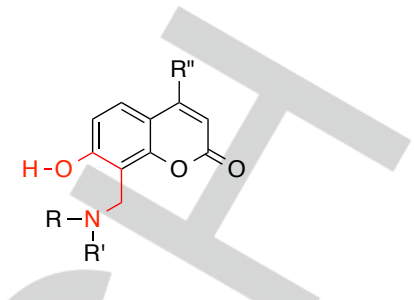

Figure 1. General structure of the coumarins found in our searches, showing in red the molecular fragment required for the potential interaction with the Asp dyad in BACE-1.

The negligible binding affinity for BChE of the initial set of AChE binders suggested that these compounds bind to the PAS in AChE, as observed for other coumarin derivatives. ${ }^{[23]}$ In order to improve binding affinity, especially for BChE, we proposed a second generation of ligands that contain an anilino group separated from the coumarin fragment by a variable length tether. While the AChE inhibitory potency remained close to the one observed in the first series, a higher BChE inhibitory potency generally resulted for these new derivatives. A final structural modification involving the $\mathrm{N}$-substitution of the 8aminomethyl moiety with a $\mathrm{N}$-benzylpiperidine, that bears a protonatable nitrogen able to establish $\pi$-cation interactions in the CAS of AChE, led to the most potent AChE inhibitor of the whole series, endowed also with moderate $B C h E$ and $A \beta$ aggregation inhibition.

\section{Results and Discussion}

The protocol for the in silico evaluation of the affinity to the chosen therapeutic targets included docking calculations for the enzymes AChE and BACE-1 and MD simulations for the binding to the $A \beta$ peptide. A summary of the methods followed in the calculations is given in the Supporting Information and for full details see reference 19 .

\section{AChE docking simulations.}

The relative small size of the first coumarins precludes their simultaneous binding to both the CAS and PAS of AChE. For this reason, we searched for binding hits through separate docking simulations focused either at the CAS or at the PAS. As seen from the results shown in Table 1 , in all cases the top scoring function displays a hit both at the CAS and PAS binding regions. In many cases, the scoring value differences between the CAS and PAS sites were rather small, although in most instances the scoring value was higher for CAS than for PAS. This outcome raised the possibility that the ligands could bind to either the CAS or the PAS. 
Table 1. Coumarin docking results to the CAS and PAS in TCAChE using ChemPLP as scoring function.

\begin{tabular}{|c|c|c|c|c|c|c|c|}
\hline Compound & $R$ & Scoring (PAS) & Scoring (CAS) & Compound & $\mathrm{R}$ & Scoring (PAS) & Scoring (CAS) \\
\hline 1 & & 87.53 & 100.57 & 15 & & 77.43 & 86.86 \\
\hline 2 & & 74.40 & 83.94 & 16 & & 72.49 & 70.91 \\
\hline 3 & & 75.04 & 84.74 & 17 & & 68.07 & 80.76 \\
\hline 4 & & 72.45 & 82.13 & 18 & & 71.89 & 70.02 \\
\hline 5 & & 76.01 & 77.70 & 19 & & 74.39 & 73.01 \\
\hline 6 & & 67.51 & 68.08 & 20 & & 76.99 & 84.77 \\
\hline 7 & & 70.95 & 83.22 & 21 & & 70.40 & 80.23 \\
\hline 8 & & 79.40 & 86.35 & 22 & & 72.90 & 71.38 \\
\hline 9 & & 73.52 & 85.15 & 23 & & 74.53 & 86.54 \\
\hline 10 & & 69.83 & 74.16 & 24 & & 66.79 & 79.46 \\
\hline 11 & & 72.06 & 77.92 & 25 & & 75.09 & 81.65 \\
\hline 12 & & 69.71 & 82.57 & 26 & & 83.42 & 82.60 \\
\hline 13 & & 75.76 & 76.59 & 27 & & 78.53 & 88.97 \\
\hline 14 & & 76.82 & 76.10 & & & & \\
\hline
\end{tabular}

In Figure 2 the resulting binding poses of compound $\mathbf{5}$ at both the CAS and PAS of TCAChE are shown. As seen from this figure, the ligand was able to make $\pi$-stacking interactions with Phe 330 and Trp 84 at the CAS and the same type of interactions with Tyr 70 and Trp 279 at the PAS.

\section{BACE-1 docking simulations.}

The results for the docking calculations of the candidate leads to BACE-1 are shown in Table S1 (see Supporting Information). As mentioned in the Methods section of the Supporting Information, the docking into BACE-1 was performed with three protein templates that differ in the degree of flap opening. As seen from table S1, there was a very limited consensus on the capabilities of this set of coumarins as BACE-1 binders. The docking calculations displayed hits only with the ChemScore function and an open flap or with the GoldScore function and a half-open flap, and only three coumarins (2, 5 and 15) out of the 27 analogues studied displayed hits for more than one scoring function or flap opening. 


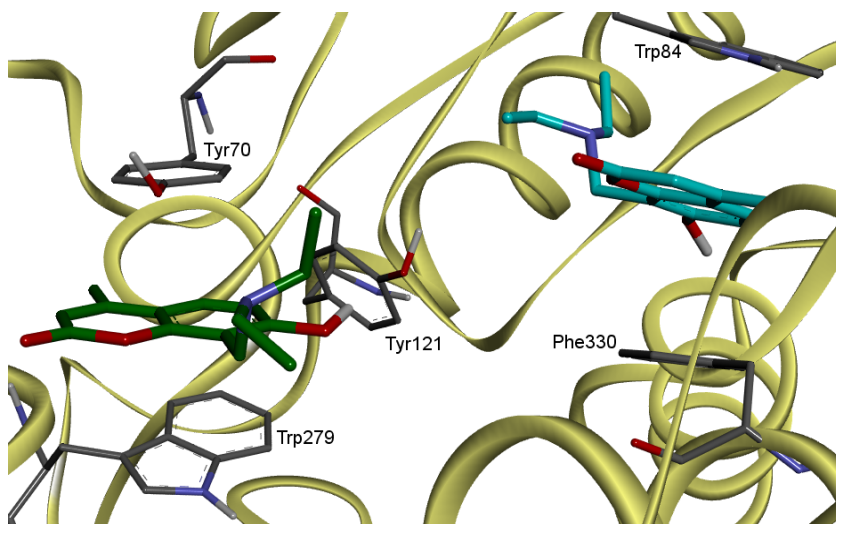

Figure 2. Top-score binding pose of compound 5 bound either to the CAS (carbons in cyan, right) or to the PAS (carbons in green, left) of TcAChE, as predicted by our docking calculations.

\section{A $\beta$ peptide binding simulations.}

In previous aggregation inhibition studies using the same MD protocol $^{[19]}$ we could rank the capability of our compounds to inhibit aggregation by evaluating their aptitude to interrupt the formation of a hairpin turn, a structural motif that has been proposed as the possible template for $A \beta$ aggregation. Figure $S 3$ (see Supporting Information) displays the $A \beta$ peptide intraresidue contact time in the presence and absence of our candidate ligands. As seen from this figure, only compound 5 precluded to some extent the formation of a beta hairpin.

Taken as a whole, our calculations predicted that the primary target of our initial set of coumarins was AChE. In order to validate our results, and eventually extend the biological activity to other AD targets we designed, synthesized and assayed a number of additional coumarins. Since previous studies ${ }^{[28-30]}$ have pointed out that the coumarin scaffold binds to the PAS of AChE we performed also BChE inhibition as well as propidium competitive binding assays (see below).

\section{Synthesis and binding assays for $A D$ targets of novel} coumarins.

Coumarin derivatives 5, 6, 10, 17, 25, 26 and 27 were prepared in moderate to good yields by Mannich condensation of commercially available 7-hydroxy-4-methylcoumarin, the appropriate amine, and formaldehyde in refluxing ethanol, as indicated in Scheme 1.

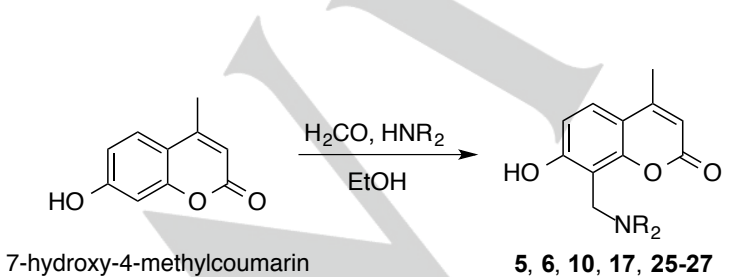

Scheme 1. Synthetic route for the coumarin derivatives.

The results from the experimental binding assays of BACE- $1,{ }^{[19]}$ inhibition of electric eel AChE (eeAChE) and horse serum BChE $(h s \mathrm{BChE}),{ }^{[4]}$ as well as the ThT $\mathrm{A} \beta$ aggregation inhibition assay, ${ }^{[48,49]}$ are shown in Table 2 . They indicate that the target to which these compounds bind the strongest is AChE, very much in support of the results of our calculations. The binding to the other targets was much weaker. For instance, our results indicated that only compounds $\mathbf{5}$ and $\mathbf{1 0}$ inhibited $A \beta$ aggregation at a level similar to 9,10-anthraquinone (nearly $30 \%$ at $100 \mu \mathrm{M}){ }^{[50]}$ The lack of substantive binding affinity for $B C h E$ should have a bearing on the AChE site to which these ligands bind. It is known that while AChE and BChE have highly homologous catalytic sites, the PAS of AChE and the corresponding binding region of $\mathrm{BChE}$ share a very low homology. For instance, the key aromatic residues Trp 279 and Tyr 70 in TcAChE are replaced in $h s \mathrm{BChE}$ by non-aromatic residues. Hence, it may be surmised that aromatic leads (like the coumarins studied in this work) that inhibit AChE but do not inhibit BChE would likely bind to the former enzyme at the PAS. Previous studies on the binding site of coumarins substituted at position 7 in AChE have also suggested that these compounds bind to the PAS. ${ }^{[28-30,51,52]}$

Since it is known that coumarin derivatives bind both MAO isoforms $^{[38-43]}$ we also performed MAO-A and MAO-B inhibition tests on rat brain mitochondrial homogenates ${ }^{[39]}$ for compounds 10, 26 and 27 and found that the inhibition was below $10 \%$ at 20 $\mu \mathrm{M}$ (data not shown).

Table 2. Biochemical assay results for the first generation of coumarin derivatives.

\begin{tabular}{ccccc}
\hline Compound & ee $\mathrm{AChE}^{[\mathrm{a}]}$ & hs BChE $^{[\mathrm{b}]}$ & $\mathrm{A \beta}(1-40)^{[\mathrm{c}]}$ & $\mathrm{BACE}^{1}{ }^{[\mathrm{d}]}$ \\
\hline $\mathbf{5}$ & $6.5 \pm 0.8$ & 0 & $20 \pm 4$ & 27.91 \\
$\mathbf{6}$ & $6.0 \pm 0.6$ & $11 \pm 1$ & $6 \pm 2$ & 23.00 \\
$\mathbf{1 0}$ & $7.9 \pm 0.7$ & 0 & $33 \pm 4$ & 23.69 \\
$\mathbf{1 7}$ & $7.7 \pm 0.1$ & 0 & 0 & 27.07 \\
$\mathbf{2 5}$ & $5.8 \pm 1.6$ & 0 & 0 & 21.13 \\
$\mathbf{2 6}$ & $7.3 \pm 1.1$ & $12 \pm 3$ & $5 \pm 4$ & 27.26 \\
$\mathbf{2 7}$ & $5.1 \pm 1.6$ & $13 \pm 4$ & $4 \pm 1$ & 21.63 \\
\hline
\end{tabular}

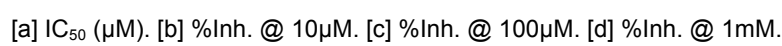

\section{Design, synthesis and binding assays of second-generation coumarins}

It is known that compounds that fit both the PAS and CAS in AChE, named dual binding site inhibitors, might include two aromatic fragments, or one aromatic and one aminic fragments, separated by a tether that should span the gorge between both sites. Indeed, previous attempts to obtain dual binding site AChE inhibitors explored charged fragments, i.e. quaternary ammonium salts and protonated amines, eventually capped by an aromatic group, at various positions of coumarins other than 8. Most of the structural variations rely on substitution at positions $3^{[30,35]}$ and $7 .{ }^{[28,29,45]}$

Since the 7-hydroxy-4-methylcoumarin moiety seemed to fit well into the PAS, we decided to generate a new set of compounds bearing an anilino motif separated by different tethers from the coumarin ring (see Scheme 2).

The Mannich condensation was carried out as before, using commercially available 7-hydroxy-4-methylcoumarin, the 
appropriate diamine and formaldehyde in refluxing ethanol. The required acyclic diamines were prepared as indicated in the Supporting Information starting from commercially available and inexpensive $\omega$-amino alcohols. The use of $N, N^{\prime}$-dimethylated diamines in the Mannich condensation afforded the expected coumarins 28-30 in good yields (Scheme 2). However, the use of primary amines ( $N$-phenylalkane-1,n-diamines where $n=2,3$, 5 ) afforded the cyclic derivatives 31-33 through a reaction with two equivalents of formaldehyde. Although it is known that primary amines react with two equivalents of formaldehyde to give the corresponding dihydrobenzoxazines, ${ }^{[53,54]}$ we observed that diamines with a spatial linker of 2 or 3 carbon atoms gave instead the corresponding imidazolidine $\mathbf{3 1}$ or perhydropyrimidine 32 , but when the two amines were separated by 5 carbon atoms the reaction favored the formation of the dihydro-1,3-oxazine 33 .

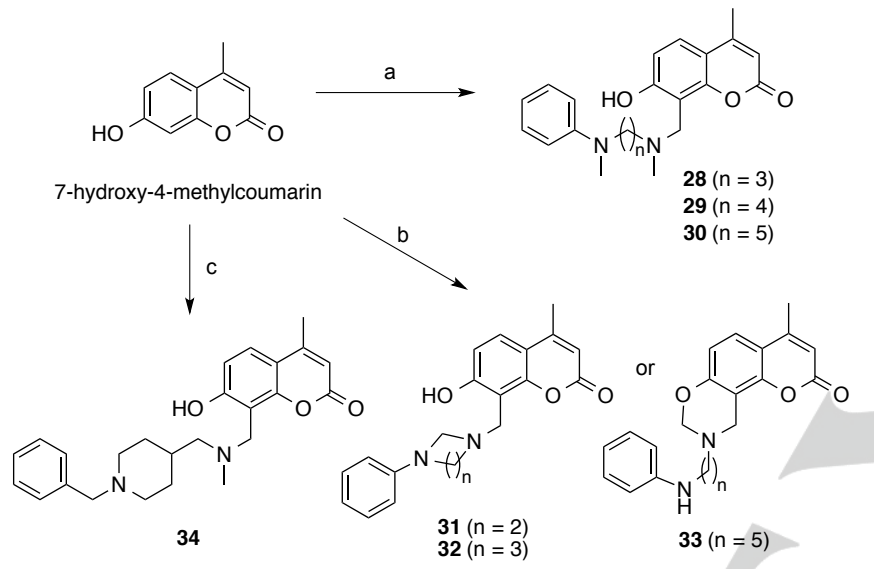

Scheme 2. Synthetic route for second and third generation coumarin derivatives. Reagents and conditions: a) $\mathrm{MeHN}\left(\mathrm{CH}_{2}\right)_{n} \mathrm{NMePh}(\mathrm{n}=3-5), \mathrm{H}_{2} \mathrm{CO}$, $\mathrm{EtOH}$, reflux, 6h; b) $\mathrm{H}_{2} \mathrm{~N}\left(\mathrm{CH}_{2}\right)_{n} \mathrm{NHPh}(\mathrm{n}=2,3,5), \mathrm{H}_{2} \mathrm{CO}$, EtOH, reflux, 6h; c) 1-(1-Benzylpiperidin-4-yl)- $\mathrm{N}$-methylmethanamine, $\mathrm{H}_{2} \mathrm{CO}$, EtOH, reflux, $6 \mathrm{~h}$.

The results of the experimental binding assays in AChE and $B C h E$, as well as the ThT A $\beta$ aggregation inhibition are shown in Table 3. As seen in this table, all compounds display affinity for $\mathrm{AChE}$, but the only compounds that displayed dual AChE-BChE affinity were some of those containing the additional annular structure (32 and $\mathbf{3 3}$ ). The only compound with an additional ring that showed lower affinity for BChE was $\mathbf{3 1}$, a result that could be related to the ring size, which was the smallest (5-membered) amongst these compounds. Compounds 28-30, structurally close to $\mathbf{3 2}$ but more flexible, bound weakly to $\mathrm{BChE}$, a result that indicated that their end anilino group (other than the coumarin moiety) did not bind efficiently to the CAS of AChE. It would seem that the additional ring in compounds 32 and 33 placed the anilino moiety in the right orientation to bind more efficiently BChE. In addition, compound $\mathbf{3 3}$ was the only compound displaying some degree of inhibition of $A \beta$ aggregation (see Table 3).
Table 3. Assay results for second and third generation candidates.

\begin{tabular}{ccccc}
\hline Compound & ee $\mathrm{AChE}^{[\mathrm{a}]}$ & $h s \mathrm{BChE}^{[\mathrm{b}]}$ & $h s \mathrm{BChE}^{[\mathrm{c}]}$ & $\mathrm{A \beta}(1-40)^{[\mathrm{d}]}$ \\
\hline $\mathbf{2 8}$ & $7.6 \pm 0.8$ & $38 \pm 3$ & -- & 0 \\
$\mathbf{2 9}$ & $7.7 \pm 0.4$ & $27 \pm 1$ & - & 0 \\
$\mathbf{3 0}$ & $5.2 \pm 0.5$ & $41 \pm 1$ & -- & 0 \\
$\mathbf{3 1}$ & $9.9 \pm 0.6$ & $41 \pm 3$ & -- & $6 \pm 2$ \\
32 & $7.2 \pm 0.5$ & -- & $5.3 \pm 0.3$ & 0 \\
33 & $6.5 \pm 0.1$ & -- & $4.7 \pm 0.4$ & $20 \pm 2$ \\
34 & $0.83 \pm 0.14$ & -- & $15 \pm 3$ & $28 \pm 4$ \\
\hline
\end{tabular}

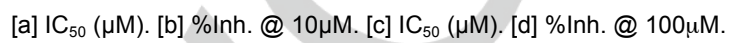

Even though our step-wise molecular design led to two interesting ChE inhibitors exhibiting micromolar affinity towards both $\mathrm{AChE}$ and $\mathrm{BChE}$, i.e. compounds 32 and 33, we designed a final molecule aiming at the recovery of a $\pi$-cation and $\pi-\pi$ interactions in the CAS of AChE and possibly of BChE. A Nbenzylpiperidine moiety, present also in the well known AChE inhibitor drug donepezil (E2020), ${ }^{[55]}$ was then attached at position 8 at an appropriate distance from the coumarin ring to yield compound $\mathbf{3 4}$ (Scheme 2). The inhibitor design (also used by other authors) ${ }^{[20,21]}$ was validated by means of perspective docking calculations on TCAChE that returned the best consensus with the largest number of hits amongst the different scoring functions.

It is worth noting that the structure of TcAChE bound to donepezil reveals a singular binding mode that differs from the ones displayed by many other potent AChE inhibitors. While a large number of AChE ligands have aromatic fragments that interact simultaneously with side chains of aromatic amino acids through a face-to-face $\pi-\pi$ interactions, the aromatic ends of donepezil interact only with one aromatic side chain in an 'open face' sandwich pose.$^{[56,57]}$ We performed docking simulations to predict binding poses of compound $\mathbf{3 4}$, using as a template the 3D structure (1EVE) of the TcAChE-donepezil complex. ${ }^{[56]}$

Figure 3 displays one of the top-score binding poses of $\mathbf{3 4}$, ranked through ChemPLP evaluation function, along with the Xray determined binding pose of donepezil. This figure displays the 'open-faced' sandwich interaction between the coumarin moiety and Trp 279 in the PAS and between the $N$-benzyl group and Trp 84 in the CAS of TCAChE, similarly to the interaction observable for donepezil. ${ }^{[56]}$ Notice that the $N$-benzyl group occupies the CAS region in accordance with our design aims.

Very satisfactorily the AChE inhibition assay fully confirmed the docking prediction as compound $\mathbf{3 4}$ resulted the most potent inhibitor of the whole series of prepared coumarins with a submicromolar $\mathrm{IC}_{50}(0.83 \mu \mathrm{M})$.

To gain further insights into the mechanism of $\mathrm{AChE}$ inhibition by compound $\mathbf{3 4}$, its kinetic of inhibition and ability to displace propidium from PAS of AChE were assayed as reported in sections below. 


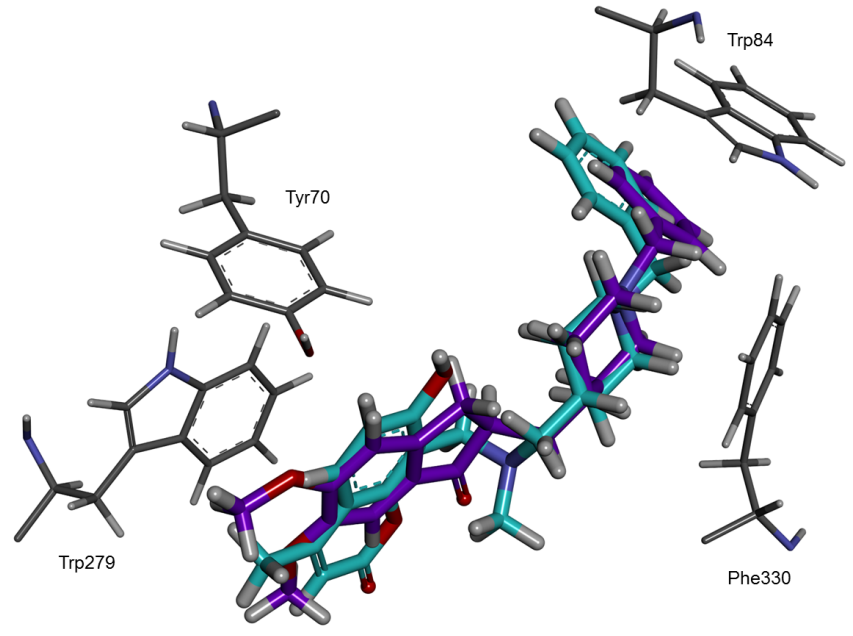

Figure 3. Binding pose of compound $\mathbf{3 4}$ (carbons in cyan) resulting from docking calculations superimposed to the X-ray crystal structure of donepezil (carbons in purple) in the TcAChE-inhibitor complex. ${ }^{[56]}$

As expected, the results from the experimental binding assays on BACE-1, carried out on compounds $\mathbf{3 1}, \mathbf{3 2}$ and $\mathbf{3 4}$, showed a low inhibition (below $20 \%$ at $100 \mu \mathrm{M}$, data not shown). MAO-A and MAO-B inhibition assays for compounds 28, 30, 31, 33 and 34 showed an inhibition below $20 \%$ at $20 \mu \mathrm{M}$.

\section{Kinetic study of the eeAChE inhibition by compound 34}

Kinetics of eeAChE inhibition were assessed by using four different concentrations of inhibitor $34(0$ to $8 \mu \mathrm{M})$ and six concentrations of substrate acetylthiocoline $(0.033$ to $0.2 \mathrm{mM})$. As expected, Lineweaver-Burk plot in Figure 4 suggested a mixed-type inhibition mechanism, typical of dual binding site inhibitors interacting at both the CAS and PAS. ${ }^{[30]}$ Measured $\mathrm{K}_{\mathrm{i}}$ for 34 was $1.43 \pm 0.25 \mu \mathrm{M}$.
Propidium displacement assay on compound 34 and other selected coumarins.

Propidium displacement assays were conducted at $100 \mu \mathrm{M}$ concentration. Propidium, is a specific ligand of PAS of $\mathrm{AChE}^{[58]}$ and its displacement indicates that the tested inhibitor binds to this site and that the higher the inhibition potency, the lower the concentration needed to displace propidium. It is worth reminding that the concentration of inhibitor necessary to displace propidium is around two orders of magnitude higher than its $I_{50}$ value. For instance, the concentration required to displace propidium by ligands with $\mathrm{IC}_{50}$ in the high nanomolar range is generally in the micromolar range.$^{[59]}$ The results from our propidium displacement assay are shown in Table 4. For a straightforward comparison among our inhibitors and donepezil, a well known dual binding site inhibitor of AChE, the percentage of propidium displacement was normalized to the maximum effect produced by donepezil $(65 \pm 2 \%$ propidium displacement in AChE). As seen in Table 4, the relative percentage of displacement of our tested coumarins was up to $89 \%$ of the maximum effect of donepezil. Compound 34 exhibited $57 \%$ of relative propidium displacement and a high displacement was observed for first generation coumarins $5(66 \%)$ or $6(69 \%)$, and even higher for 17 (89\%) or 26 (82\%). High relative displacement values were measured also for second generation coumarins with a few exceptions, e.g. compound 28 (34\%).

Table 4. Percentage of propidium displacement by coumarin candidates normalized to the maximum effect of donepezil.

\begin{tabular}{cccc}
\hline Compound & \%Disp @100 $\mu \mathrm{M}$ & Compound & \%Disp @100 $\mu \mathrm{M}$ \\
\hline $\mathbf{5}$ & $66.24 \pm 1.06$ & $\mathbf{2 8}$ & $33.78 \pm 2.39$ \\
$\mathbf{6}$ & $69.03 \pm 4.52$ & $\mathbf{2 9}$ & $48.89 \pm 0.59$ \\
$\mathbf{1 0}$ & $63.93 \pm 3.32$ & $\mathbf{3 0}$ & $52.95 \pm 4.84$ \\
$\mathbf{1 7}$ & $89.05 \pm 3.97$ & $\mathbf{3 1}$ & $42.61 \pm 1.63$ \\
$\mathbf{2 5}$ & $58.85 \pm 1.99$ & $\mathbf{3 2}$ & $54.03 \pm 1.27$ \\
$\mathbf{2 6}$ & $81.98 \pm 3.30$ & $\mathbf{3 3}$ & $56.41 \pm 4.29$ \\
$\mathbf{2 7}$ & $54.22 \pm 0.21$ & $\mathbf{3 4}$ & $56.99 \pm 1.20$ \\
\hline
\end{tabular}

\section{Conclusions}

A search based on a multitarget pharmacophore previously proposed ${ }^{[19]}$ has enabled us to find a number of 8-aminomethyl7-hydroxy-4-methyl coumarins as suitable scaffolds that could fulfill our polypharmacology lead search against $A D$. These compounds contain some of the structural characteristics that could make them good binders for amyloid cascade targets as well as for cholinergic targets. For instance the hydroxyl and amino groups could anchor the molecule to the Asp dyad in BACE-1, while the coumarin ring could bind to the PAS of AChE. The resulting leads (with a large variety of aminomethyl substituents at position 8) have a substitution pattern that has been rarely explored for ChE inhibition, ${ }^{[60]}$ and never as BACE-1 or aggregation inhibitors. A protocol previously developed in our 
laboratory was applied to determine which $A D$ therapeutic targets could have been modulated by these compounds. The results of our simulations predicted that these compounds present a consensus only as ChE binders, an outcome validated by enzyme inhibition assays on ChE and BACE-1 and inhibition assay of $A \beta$ aggregation assessed by the ThT fluorescence method. ${ }^{[49]}$ This outcome provides further proof for the reliability of our computer aided protocol ${ }^{[19]}$ for the search of multitarget leads.

Given their size, our inhibitors could bind either the PAS or CAS sites in AChE, forming $\pi$-cation and $\pi-\pi$ interactions with key aromatic residues. Many of the exit poses indicated that the scoring functions did not differentiate between the two binding sites. Previous studies with a different 7-hydroxycoumarin, that is the 3-chloro-7-hydroxy-4-methylcoumarin, demonstrated that this inhibitor bind to the PAS of AChE. Only when some key aromatic residues of the PAS were mutated to non-aromatic ones, the coumarin was able to bind to the CAS. ${ }^{[52]}$

To generate dual binding site inhibitors we proceeded to enlarge the coumarin substituent at position 8 , capping it with anilino and finally with a benzylamino moiety. The synthesis led to two groups of compounds that differentiate themselves in the presence or absence of an additional ring (see Scheme 2). Compound 34 was the candidate that displayed the highest binding affinity for AChE reaching a sub-micromolar $\mathrm{IC}_{50}(0.83$ $\mu \mathrm{M})$, while keeping a $\mu \mathrm{M}$ affinity for $\mathrm{BChE}$. This compound bears some of the traits of donepezil (Aricept or E2020), ${ }^{\left[{ }^{11]}\right.}$ one of the drugs used in clinic for the palliative treatment of $A D$. As donepezil, inhibitor 34 contains an $\mathrm{N}$-benzylpiperidine fragment and shares a similar binding mode at the PAS and CAS of AChE as illustrated in Figure 3. Kinetic studies suggested a mixed type mechanism of inhibition indicating that compound 34 could be a dual binding site inhibitor as donepezil.

Our inhibition data would indicate that the presence of the additional ring is essential for the binding at BChE with affinities similar to that of AChE (see Table 3). As mentioned before, the only exception was compound $\mathbf{3 1}$, a weak BChE binder, since it contains an imidazolidine group instead of the larger hexahydropyrimidine (present in 32). Finally, compound 34 could be considered as a promising lead for further structural modifications aimed at improving its inhibition potency at AChE and expanding its activity towards other therapeutic targets of amyloid cascade, such as BACE-1 and $A \beta$-aggregation.

Supporting Information. Computational methodology details as well as experimental procedures for the syntheses and in vitro biological evaluation of compounds listed in Tables 2 and 3.

\section{Acknowledgements}

Financial support from the Ministerio de Economia $y$ Competitividad of Spain (Project CTQ2014-55208-P) and the Xunta de Galicia (10CSA209063PR and GRC2014/029) is gratefully acknowledged. The Italian authors thank the University of Bari for partial financial support (Fondi di Ateneo 2014-2015). We also would like to thank the Center for Supercomputing in Galicia (CESGA) for computer time.
Keywords: Alzheimer's disease • 8-aminomethyl-7-hydroxy-4methylcoumarins $\cdot$ cholinesterases inhibition $\cdot$ computational chemistry $\bullet$ drug design $\cdot$ multitarget leads

\section{References:}

[1] F. Mangialasche, A. Solomon, B. Winblad, P. Mecocci, M. Kivipelto Lancet Neurol. 2010, 9, 702-716.

[2] C. Berck, M. N. Sabbagh, Drugs Aging 2013, 30, 783-792.

[3] J. Hardy, D. J. Selkoe, Science 2002, 297, 353-356.

[4] D. M. Walsh, D. J. Selkoe, Neuron 2004, 44, 181-193.

[5] D. J. Selkoe, Science 2012, 337, 1488-1492.

[6] J. Brouillette, Curr. Pharm. Des. 2014, 20, 2506-2519.

[7] N. C. Inestrosa, A. Alvarez, C. A. Pérez, R. D. Moreno, M. Vicente, C. Linker, O. I. Casanueva, C. Soto, J. Garrido, Neuron 1996, 16, 881-891.

[8] T. Thomas, Neurobiol. Aging 2000, 21, 343-348.

[9] Z. Cai, Mol. Med. Rep. 2014, 9, 1533-1541.

[10] L. Pisani, M. Catto, F. Leonetti, O. Nicolotti, A. Stefanachi, F. Campagna, A. Carotti, Curr. Med. Chem. 2011, 18, 4568-4587.

[11] N. Guzior, A. Wieckowska, D. Panek, B. Malawska, Curr. Med. Chem. 2015, 22, 373-404.

[12] A. Cavalli, M. L. Bolognesi, S. Capsoni, V. Andrisano, M. Bartolini, E. Margotti, A. Cattaneo, M. Recanatini, C. Melchiorre, Angew. Chem. Int. Ed. 2007, 46, 3689-3692.

[13] Y. Zhu, K. Xiao, L. Ma, B. Xiong, Y. Fu, H. Yu, W. Wang, X. Wang, D. Hu, H. Peng, J. Li, Q. Gong, Q. Chai, X. Tang, H. Zhang, J. Li, J. Shen, Bioorg. Med. Chem. 2009, 17, 1600-1613.

[14] M. I. Fernández-Bachiller, C. Pérez, L. Monjas, J. Rademann, M. I. Rodríguez-Franco, J. Med. Chem. 2012, 55, 1303-1317.

[15] S. S. Xie, X. Wang, N. Jiang, W. Yu, K. D. Wang, J. S. Lan, Z. R. Li, L. Y. Kong, Eur. J. Med. Chem. 2015, 95, 153-165.

[16] M. L. Bolognesi, A. Cavalli, L. Valgimigli, M. Bartolini, M. Rosini, V. Andrisano, M. Recanatini, C. Melchiorre, J. Med. Chem. 2007, 50, 6446-6449.

[17] M. L. Bolognesi, M. Bartolini, A. Tarozzi, F. Morroni, F. Lizzi, A. Milelli, A. Minarini, M. Rosini, P. Hrelia, V. Andrisano, C. Melchiorre, Bioorg. Med. Chem. Lett. 2011, 21, 2655-2658.

[18] R.-S. Li, X.-B. Wang, X.-J. Hu, L.-Y. Kong, Bioorg. Med. Chem. Lett. 2013, 23, 2636-2641.

[19] J. L. Domínguez, F. Fernández-Nieto, M. Castro, M. Catto, M. R. Paleo, S. Porto, F. J. Sardina, J. M. Brea, A. Carotti, M. C. Villaverde, F. Sussman, J. Chem. Inf. Model. 2015, 55, 135-148.

[20] F. Prati, C. Bergamini, R. Fato, O. Soukup, J. Korabecny, V. Andrisano, M. Bartolini, M. L. Bolognesi, Chem. Med. Chem. 2016, 11, 0000-0000. DOI: $10.1002 / \mathrm{cmdc} .201600014$.

[21] I. Bolea, J. Juárez-Jiménez, C. de los Ríos, M. Chioua, R. Pouplana, F. J. Luque, M. Unzeta, J. Marco-Contelles, A. Samadi, J. Med. Chem. 2011, 54, 8251-8270.

[22] E. Viayna, I. Sola, M. Bartolini, A. De Simone, C. Tapia-Rojas, F. G. Serrano, R. Sabaté, J. Juárez-Jiménez, B. Pérez, F. J. Luque, V. Andrisano, M. V. Clos, N. C. Inestrosa, D. Muñoz-Torrero, J. Med. Chem. 2014, 57, 2549-2567.

[23] P. Anand, B. Singh, N. Singh, Bioorg. Med. Chem. 2012, 20, $1175-$ 1180.

[24] C. R. Wu, C. L. Chang, P. Y. Hsieh, L. W. Lin, H. Ching, Planta Med. 2007, 73, 275-278.

[25] I. Najmanová, M. Doseděl, R. Hrdina, P. Anzenbacher, T. Filipský, M. Řiha, P. Mladěnka, Curr. Top. Med. Chem. 2015, 15, 830-849.

[26] K. Skalicka-Woźniak, I. E. Orhan, G. A. Cordell, S. M. Nabavi, B. Budzyńska, Pharmacol. Res. 2016, 103, 188-203.

[27] A. Thakur, R. Singla, V. Jaitak, Eur. J. Med. Chem. 2015, 101, 476-495

[28] L. Pisani, M. Catto, I. Giangreco, F. Leonetti, O. Nicolotti, A. Stefanachi, S. Cellamare, A. Carotti, Chem. Med. Chem. 2010, 5, 1616-1630.

[29] F. Leonetti, M. Catto, O. Nicolotti, L. Pisani, A. Cappa, A. Stefanachi, A. Carotti, Bioorg. Med. Chem. 2008, 16, 7450-7456.

[30] M. Catto, L. Pisani, F. Leonetti, O. Nicolotti, P. Pesce, A. Stefanachi, S Cellamare, A. Carotti, Bioorg. Med. Chem. 2013, 21, 146-152. 
[31] S. F. Razavi, M. Khoobi, H. Nadri, A. Sakhteman, A. Moradi, S. Emami, A. Foroumadi, A. Shafiee, Eur. J. Med. Chem. 2013, 64, 252-259.

[32] M. Alipour, M. Khoobi, A. Moradi, H. Nadri, F. H. Moghadam, S. Emami, Z. Hasanpour, A. Foroumadi, A. Shafiee, Eur. J. Med. Chem. 2014, 82 , 536-544.

[33] S. Hamulakova, L. Janovec, M. Hrabinova, K. Spilovska, J. Korabecny, P. Kristian, K. Kuca, J. Imrich, J. Med. Chem. 2014, 57, 7073-7084.

[34] D. D. Soto-Ortega, B. D. Murphy, F. J. Gonzalez-Velasquez, K. A Wilson, F. Xie, Q. Wang, M. A. Moss, Bioorg. Med. Chem. 2011, 19 2596-2602.

[35] A. Tarozzi, M. Bartolini, L. Piazzi, L. Valgimigli, R. Amorati, C. Bolondi, A. Djemil, F. Mancini, V. Andrisano, A. Rampa, Pharmacol Res. Perspect. 2014, 2, e00023.

[36] C. Garino, N. Pietrancosta, Y. Laras, V. Moret, A. Rolland, G. Quéléver, J.-L. Kraus, Bioorg. Med. Chem. Lett. 2006, 16, 1995-1999.

[37] P. O. Patil, S. B. Bari, S. D. Firke, P. K. Deshmukh, S. T. Donda, D. A. Patil, Bioorg. Med. Chem. 2013, 21, 2434-2450.

[38] C. Gnerre, M. Catto, F. Leonetti, P. Weber, P.-A. Carrupt, C. Altomare, A. Carotti, B. Testa, J. Med. Chem. 2000, 43, 4747-4758.

[39] M. Catto, O. Nicolotti, F. Leonetti, A. Carotti, A. D. Favia, R. Soto-Otero, E. Mendez-Alvarez, A. Carotti, J. Med. Chem. 2006, 49, 4912-4925.

[40] A. Carotti, C. Altomare, M. Catto, C. Gnerre, L. Summo, A. De Marco, S. Rose, P. Jenner, B. Testa, Chem. Biodivers. 2006, 3,134-49.

[41] L. Pisani, G. Muncipinto, T. F. Miscioscia, O. Nicolotti, F. Leonetti, M. Catto, C. Caccia, P. Salvati, R. Soto-Otero, E. Mendez-Alvarez, C Passeleu, A. Carotti, J. Med. Chem. 2009, 52, 6685-6706.

[42] L. Pisani, M. Catto, O. Nicolotti, G. Grossi, M. Di Braccio, R. Soto-Otero E. Mendez-Alvarez, A. Stefanachi, G. Gadaleta, A. Carotti, Eur. J. Med. Chem. 2013, 70, 723-739.

[43] L. Pisani, R. Farina, O. Nicolotti, D. Gadaleta, R. Soto-Otero, M. Catto, M. Di Braccio, E. Mendez-Alvarez, A. Carotti, Eur. J. Med. Chem. 2015, 89, 98-105.

[44] C. Bruehlmann, F. Ooms, P. A. Carrupt, B. Testa, M. Catto, F. Leonetti, C. Altomare, A. Carotti, J. Med. Chem. 2001, 44, 3195-3198.

[45] R. Farina, L. Pisani, M. Catto, O. Nicolotti, D. Gadaleta, N. Denora, R. Soto-Otero, E. Mendez-Alvarez, C. S. Passos, G. Muncipinto, C. D Altomare, A. Nurisso, P.-A. Carrupt, A. Carotti, J. Med. Chem. 2015, 58, 5561-5578.

[46] M. Mazzei, E. Nieddu, M. Miele, A. Balbi, M. Ferrone, M. Fermeglia, M. T. Mazzei, S. Pricl, P. La Colla, F. Marongiu, C. Ibba, R. Loddo, Bioorg Med. Chem. 2008, 16, 2591-2605.

[47] G. L. Ellman, K. D. Courtney, V. Andres Jr., R. M. Feartherstone, Biochem. Pharmacol. 1961, 7, 88-95.

[48] H. LeVine III, Protein Sci. 1993, 2, 404-410.

[49] S. Cellamare, A. Stefanachi, D. A. Stolfa, T. Basile, M. Catto, F. Campagna, E. Sotelo, P. Acquafredda, A. Carotti, Bioorg. Med. Chem. 2008, 16, 4810-4822.

[50] M. Convertino, R. Pellarin, M. Catto, A. Carotti, A. Caflisch, Protein Sci. 2009, 18, 792-800.

[51] Z. Radic, E. Reiner, P. Taylor, Mol. Pharmacol. 1991, 39, 98-104

[52] V. Simeon-Rudolf, Z. Kovarik, Z. Radic, E. Reiner, Chem. Biol. Interact. 1999, 119-120, 119-128.

[53] R. B. Desai, J. Org. Chem. 1961, 26, 5251-5253.

[54] M. Tramontini, Synthesis 1973, 703-775.

[55] H. Sugimoto, Y. limura, Y. Yamanishi, K. Yamatsu, K. J. Med. Chem. 1995, 38, 4821-4829.

[56] G. Kryger, I. Silman, J. L. Sussman, Structure 1999, 7, 297-307.

[57] J. Cheung, M. J. Rudolph, F. Burshteyn, M. S. Cassidy, E. N. Gary, J. Love, M. C. Franklin, J. J. Height, J. Med. Chem. 2012, 55, 10282 10286.

[58] P. Taylor, J. Lwebuga-Mukasa, S. Lappi, J. Rademacher, Mol. Pharmacol. 1974, 10, 703-708.

[59] D. Alonso, I. Dorronsoro, L. Rubio, P. Muñoz, E. García-Palomero, M. Del Monte, A. Bidon-Chanal, M. Orozco, F. J. Luque, A. Castro, M Medina, A. Martínez, Bioorg. Med. Chem. 2005, 13, 6588-6597.

[60] F. L. Vilela, J. I. da Silva, L. C. Curcino Vieira, G. C. R. Bernasconi, A Gonçalves Corrêa, Q. Bezerra Cass, C. L. Cardoso, J. Chromatogr. B 2014, 968, 87-93.
[61] Y. Kawakami, A. Inoue, T. Kawai, M. Wakita, H. Sugimoto, A. J. Hopfinger, Bioorg. Med. Chem. 1996, 4, 1429-1446.

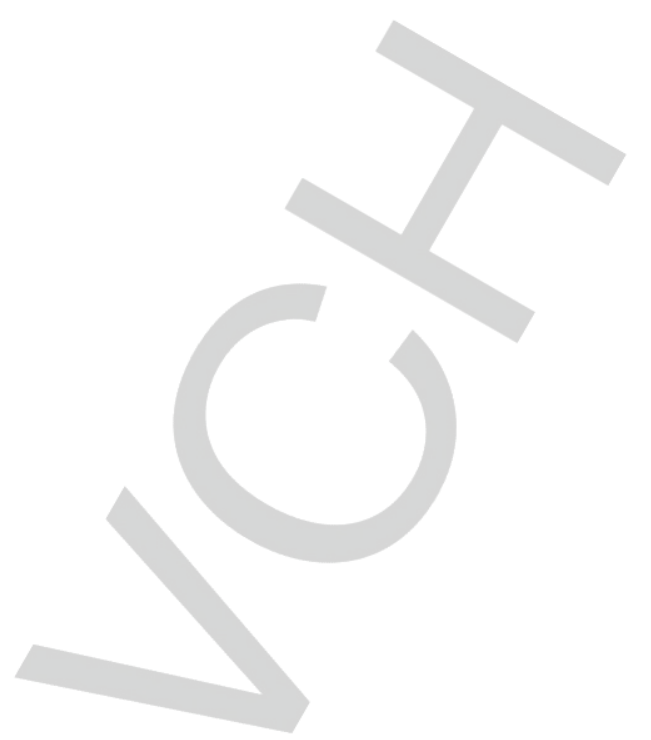




\section{Entry for the Table of Contents}

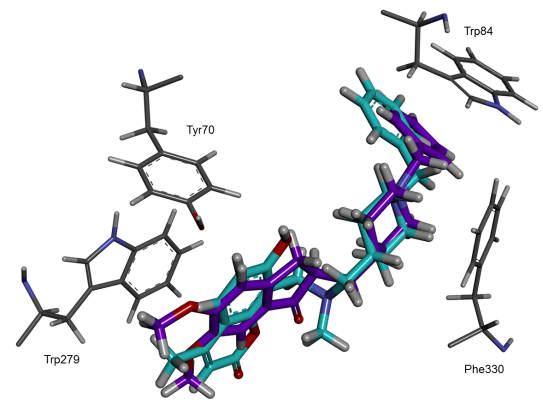

Application of a computer protocol to the search of multitarget leads for Alzheimer's disease led us to coumarine derivatives with a scantily studied susbtitution pattern that are dual site binders of AChE and also inhibit BChE and amyloid aggregation. 